\title{
Evaluación de la capacidad de absorción de energía de impacto y dureza en probetas impresas en 3D de PLA y ABS con estructura cúbica y tri hexagonal.
}

\section{Evaluation of the impact energy absorption capacity and hardness in $3 D$ printed specimens of PLA and ABS with cubic and tri-hexagonal structure.}

Miguel Ángel Escobar Guachambala. ${ }^{1}$, Javier José Gavilanes Carrión. ${ }^{2}$ \& Mesías Heriberto Freire Quintanilla. ${ }^{3}$

\section{Recibido:15-01-2020 / Revisado: 06-02-2020 /Aceptado: 08-03-2020/ Publicado: 04-04-2020}

\begin{abstract}
.
DOI: https://doi.org/10.33262/concienciadigital.v3i2.1204

Currently, custom orthotics are being built for physical rehabilitation using 3D rapid prototyping, due to this it is important to know their mechanical resistance, due to it has been proposed to test 3D printed specimens with PLA and ABS filaments. The test specimens were designed using CAD software, based on ASTM D256; Later they were 3D printed with a cubic and tri-hexagonal filling structure in PLA and ABS materials. Several tests were carried out to analyze the resistance of the test pieces: the Izod pendulum impact test, microscopy of the breaking surface and the SHORE D hardness analysis. Based on the results obtained, it was determined that the 3D printed specimen with ABS offers greater absorption of impact energy with respect to the PLA specimen; The filling structure of the test piece that gives the greatest mechanical resistance is the cubic structure compared to the tri-hexagonal structure. Furthermore, it is determined that the hardness of the PLA specimen is greater than that of the ABS specimen, finally it was observed that the fracture of the PLA specimen is linear, while the fracture of the ABS specimen is zigzag shaped.
\end{abstract}

Keywords: 3D printing, Shore D, Izod impact.

\section{Resumen.}

En la actualidad se construyen ortesis personalizadas para rehabilitación física mediante prototipado rápido $3 \mathrm{D}$, debido a esto es de importancia conocer su resistencia mecánica, por esto

\footnotetext{
${ }^{1}$ Escuela Superior Politécnica de Chimborazo, maescobar@espoch.edu.ec

${ }^{2}$ Escuela Superior Politécnica de Chimborazo, javier.gavilanes@espoch.edu.ec

3 Escuela Superior Politécnica de Chimborazo, mefreire@espoch.edu.ec
} 
se plantea ensayar probetas impresas en 3D con filamentos de PLA y ABS. Las probetas de estudio se diseñaron mediante software CAD, en función de la norma ASTM D256; después se imprimieron en 3D con estructura de relleno cúbica y tri hexagonal en materiales de PLA y ABS. Para el análisis de resistencia de las probetas se desarrolló varios ensayos: el ensayo de impacto con péndulo tipo Izod, microscopia de la superficie de rotura y el análisis de dureza SHORE D. En función de los resultados obtenidos se determinó que la probeta impresa en 3D con ABS ofrece mayor absorción de energía de impacto con respecto a la probeta de PLA; la estructura de relleno de la probeta que da mayor resistencia mecánica es la estructura cubica comparado con la estructura tri hexagonal. Además se determina que la dureza de la probeta de PLA es mayor que la probeta de $\mathrm{ABS}$, finalmente se observó que la fractura de la probeta de PLA es lineal, mientras que la fractura de la probeta de ABS es tiene una forma de zigzag.

Palabras claves: Impresión en 3D, Shore D, impacto Izod.

\section{Introducción.}

Durante el desarrollo del diseño y prototipado de ortesis de rehabilitación física, como por ejemplo férulas, prototipos de brazo, equipos de rehabilitación de tobillo, exoesqueletos, la mayoría de sus partes personalizadas están en función de la antropometría de cada persona (Arce, 2005); por lo cual el método de impresión 3D mediante aporte de polímero fundido es un método rápido y de bajo costo, los materiales comúnmente utilizados son filamentos poliméricos de ABS y PLA (Reyes, n.d.).

Con el avance de la tecnología es posible disponer de impresoras 3D para polímero fundido a un bajo costo y con mayor precisión, mediante el uso de esta tecnología de punta en prototipado rápido se puede obtener equipos de rehabilitación física personalizados de acuerdo al requerimiento de cada cliente. (Torres et al., 2012).

Las partes de equipos para rehabilitación u otro tipo de máquinas en condiciones de funcionamiento están expuestas a impactos, rayaduras, roturas, como consecuencias de choques, golpes, caídas accidentales y por diferentes condiciones de trabajo, por lo cual es importante analizar la capacidad de absorber energía de impacto, dureza y estudio de la microestructura en la zona de fractura (Molina Osejos, 2016). Por lo cual es de importancia conocer el comportamiento de la resistencia mecánica de productos impresos en 3D que son implementados en equipos para generar un trabajo útil (Tovar et al., 2018).

\section{Péndulo de impacto tipo Izod}

Entre los métodos para la determinación de la resistencia al impacto de los plásticos se establece el análisis de impacto tipo Izod según lo establecido en la norma ASTM D256 para polímeros. Las máquina de impacto tipo Izod tienen un martillo tipo péndulo, su arquitectura esta estandarizada para cumplir con los lineamientos de una determinada norma, durante el desarrollo del ensayo la altura fija de caída del brazo oscilante determina la velocidad de la carga (martillo) en el momento 
del impacto . Sin embargo, los péndulos oscilantes tienen diferentes energías iniciales producidas al variar sus pesos efectivos, los pesos de los martillos a usar están en función de las muestras a ensayar, debido a la diferente resistencia al impacto que ofrece cada material en específico. (Cardona et al., 2016)

Figura 1. Péndulo tipo Izod para polímeros.

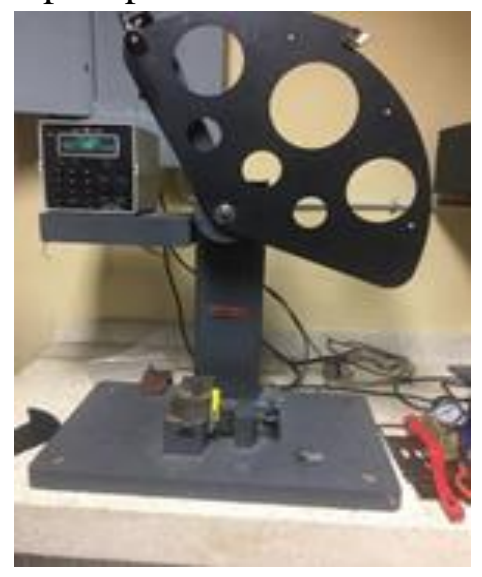

Fuente: Elaboración propia.

\section{Impresión 3D con polímero fundido.}

La impresión 3D por aporte de polímero fundido, se basa en que un objeto tridimensional es creado mediante el aporte capas sucesivas de material de polímero fundido, con una sección circular dependiendo del diámetro del extrusor; los materiales comúnmente utilizados a nivel local son ABS y PLA (Sin, 2012), debido a su bajo costo y disponibilidad.

La impresión en 3D es el método más rápido para construir prototipos de equipos y máquinas, con bajo costo. Los costos de las impresoras en estarán relacionan de su precio de adquisición y la tolerancia dimensional de los productos construidos (León \& Marcos-Fernández, 2019).

Figura 2. Impresión 3D en polímeros

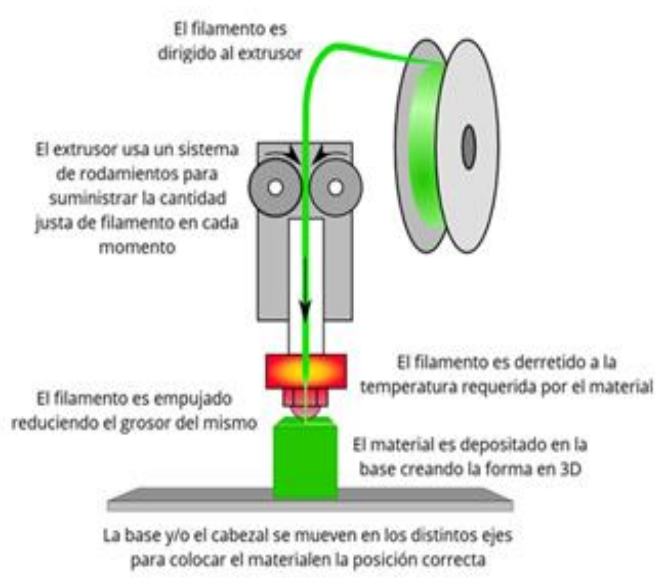

Fuente: Elaboración propia. 


\section{Ensayo de impacto Izod}

La prueba mide la resistencia al impacto de un péndulo oscilante. El ensayo de impacto Izod se define como la energía cinética necesaria para iniciar la fractura y continuar la fractura hasta que la muestra se rompe. Las muestras para ensayo Izod tienen muescas para evitar la deformación de la probeta en caso de impacto. Esta prueba se puede utilizar como una verificación de control de calidad rápida y fácil para determinar si el material es normal (Roberson et al., 2015).

Procedimiento de prueba: La probeta se sujeta al péndulo. El martillo se libera y golpea en la muesca. Si no se rompe, se usa un martillo con mayor masa hasta que ocurra una falla por impacto. Dado que muchos materiales (especialmente los termoplásticos) muestran una menor resistencia al impacto a temperaturas reducidas, a veces es apropiado ensayar los materiales a temperaturas que simulen el entorno de trabajo real.

Tamaño de la muestra: La probeta estándar para ASTM es de 64 x 12,7 x 3,2 mm (21/2 x 1/2 x 1/8 de pulgada). El grosor de la muestra más común es de 3,2 mm (0,125 pulgadas), pero el grosor preferido es de $6,4 \mathrm{~mm}$ (0,25 pulgadas) porque no es tan probable que se doble o aplaste. La profundidad debajo de la muesca de la muestra es de $10.2 \mathrm{~mm}$ (0.4 pulgadas).

Valores: La energía absorbida de impacto de ASTM se expresa en J/m o ft-lb/in. La resistencia al impacto se calcula dividiendo la energía del impacto en $\mathrm{J}$ (o ft-lb) por el ancho de la muestra que es igual $12.7 \mathrm{~mm}$. El resultado de la prueba es típicamente el promedio de 5 muestras.

Fuente: Elaboración propia.
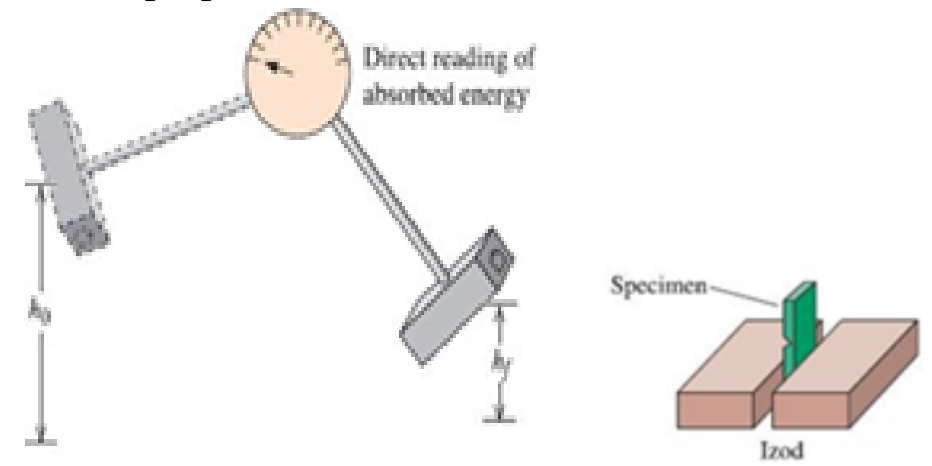

Figura 3. Esquema ensayo de impacto Izod.

La ecuación para determinar la energía usada para romper se expresa en la siguiente ecuación:

$$
T=P\left(h_{0}-h_{f}\right) g=P l(\cos \beta-\cos \alpha) g
$$

\section{Donde:}

$T$ : es la energía usada para romper la probeta 
$P$ :es la masa del péndulo medida en kilogramos

Ho: es la altura

inicial

$h f$ : es la altura final

l: es la longitud del péndulo medida en metros

$\alpha$ : es el ángulo inicial

$\beta$ :es el ángulo final.

\section{Microscopia - SEM}

El microscopio electrónico de barrido (SEM, Scanning Electron Microscopy), es un microscopio electrónico de barrido compacto y versátil, usa electrones en lugar de luz para formar imágenes de alta resolución, detalles espacialmente cercanos en la muestra pueden ser examinados a muchos aumentos, permitiendo una aproximación profunda al mundo atómico en materiales pétreos, metálicos, orgánicos, etc.

Este SEM se utiliza en una amplia gama de campos, como la biotecnología y la nanotecnología, cubriendo diversas aplicaciones, desde el desarrollo de materiales, pruebas, evaluación y análisis de defectos hasta el control de calidad, etc. Con un modelo integrado en EDS, un sistema de software fácil de usar permite una integración fluida de observación, análisis elemental y generación de informes.

Fuente: Laboratorio de Materiales -ESPOCH

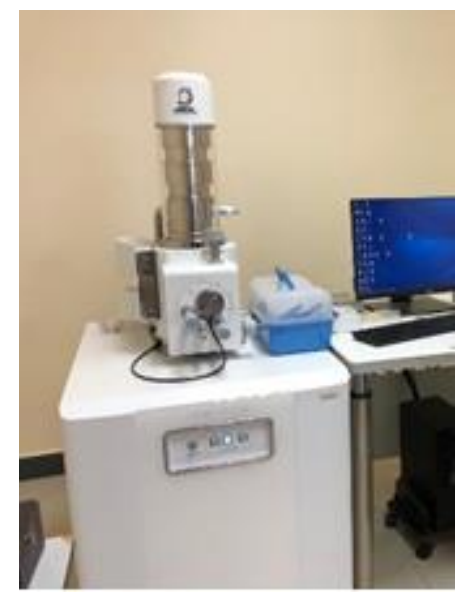

Figura 4. SEM JSM-IT100

\section{Ensayo de dureza tipo shore $D$.}

La determinación de la dureza de probetas impresas en 3D con polímeros, se realiza con un ensayo de dureza tipo Shore D según la escala para ensayos en plásticos, que consiste en un 
procedimiento sencillo: se presiona el contra el objeto de ensayo un identador metálico endurecido sostenido por un resorte con una forma definida. Según la profundidad de la impresión, dicho material será más o menos duro (Mix \& Giacomin, 2011).

Fuente: Laboratorio Materiales ESPOCH

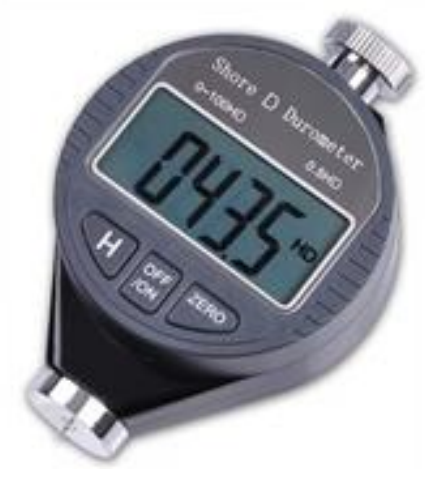

Figura 5. Durómetro Shore D.

En la siguiente tabla se muestra los valores de dureza en polímeros (Ramos et al., 2015).

Tabla 1. Rango de dureza Shore para polímeros.

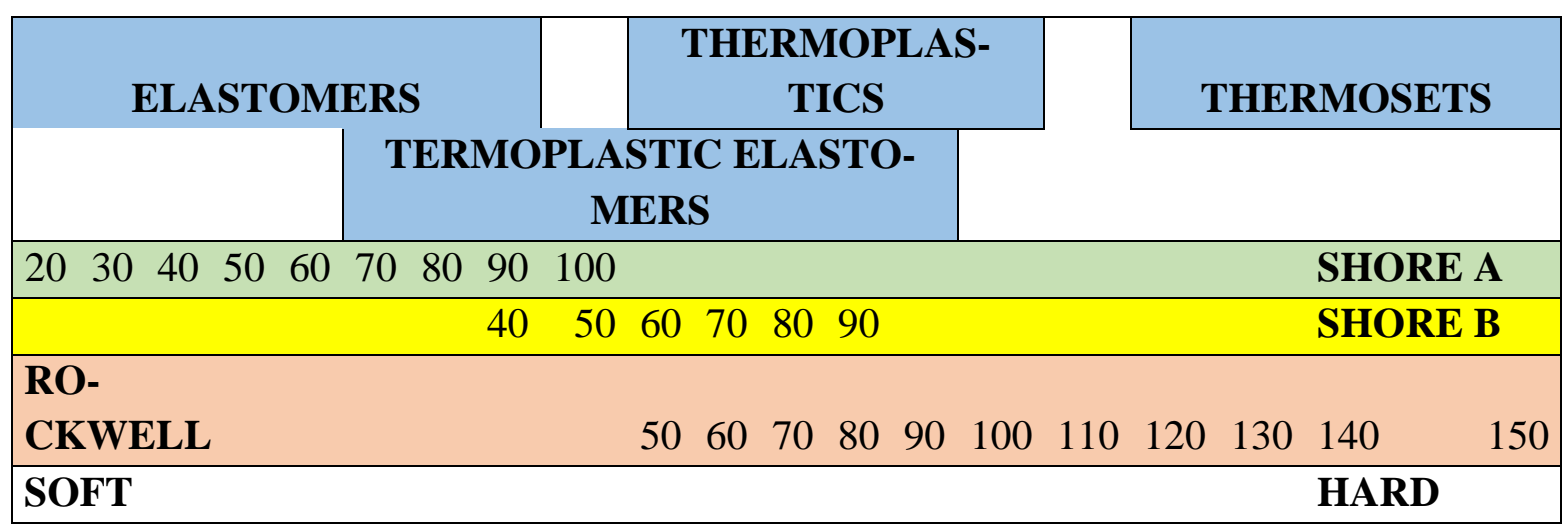

Fuente: Zwick Roel, 2020

\section{Metodología}

En la figura se resume los ensayos para determinar la capacidad de absorber energía de impacto, dureza y microscopia de la zona de fractura en probetas impresas en 3D con ABS y PLA. 
Figura 6. Metodología para ensayos en probetas impresas en 3D

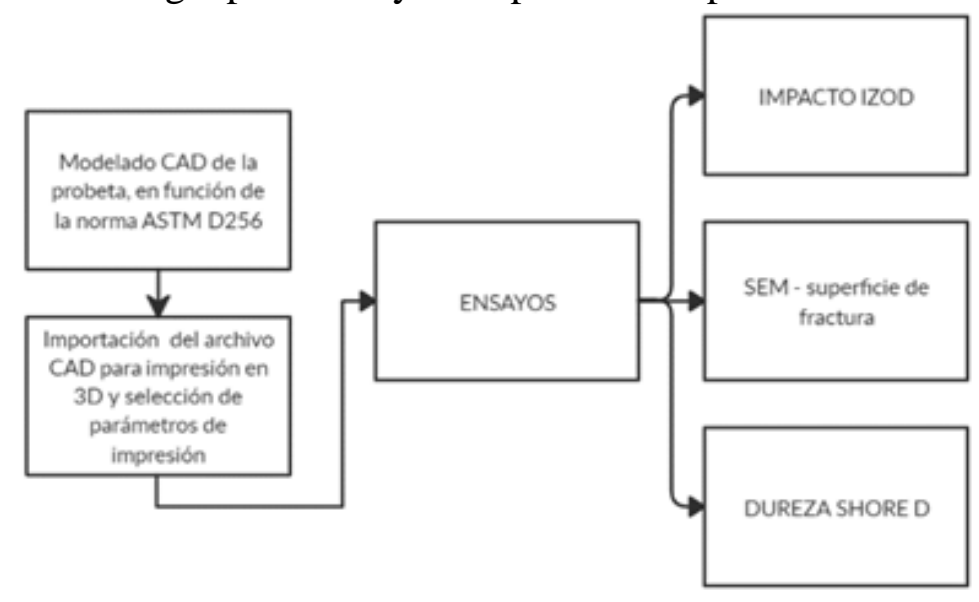

Fuente: Elaboración propia.

\section{Diseño y prototipado rápido.}

El primer requerimiento de la investigación es obtener probetas impresas en 3D, para lo cual se inicia con el diseño de las probetas en un software CAD en función de la norma ASTM D256; en base a estos diseños, se importa la información al software de impresión 3D, a continuación se muestra la geometría de la probeta.

Figura 7. Probeta para impresión 3D

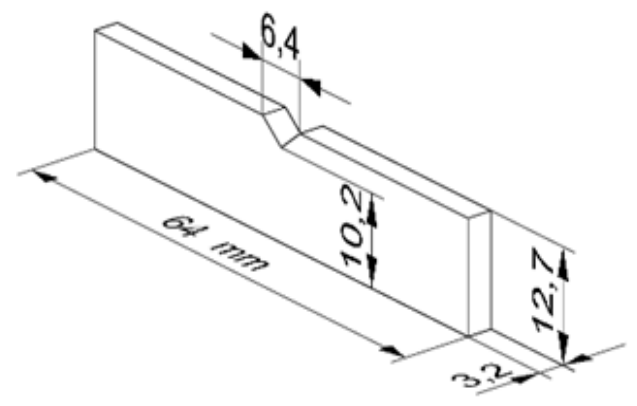

Fuente: Elaboración propia.

En la siguiente figura se observa una estructura cubica $80 \%$ de relleno.

Figura 8. Estructura cubica $80 \%$ de relleno.

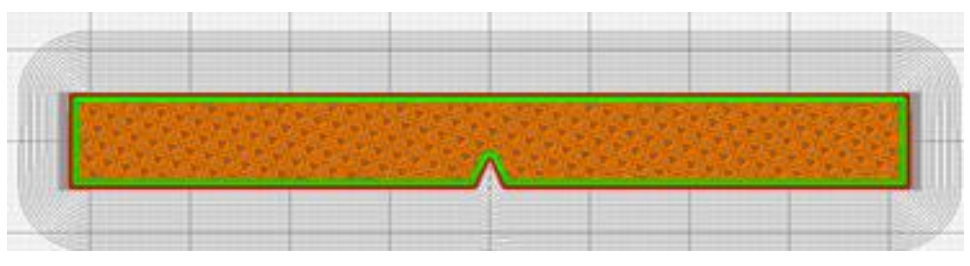

Fuente: Elaboración propia. 
En la siguiente figura se observa una estructura tri-hexagonal $80 \%$ de relleno.

Figura 9. Estructura tri-hexagonal $80 \%$ de relleno.

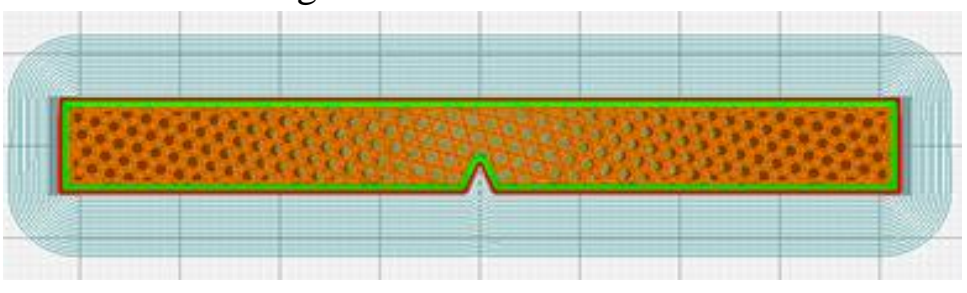

Fuente: Elaboración propia.

En la figura se observa la secuencia de procesos para la impresión de las probetas para el ensayo IZOD.|

Figura 10. Impresión 3D de probetas para el ensayo de impacto.
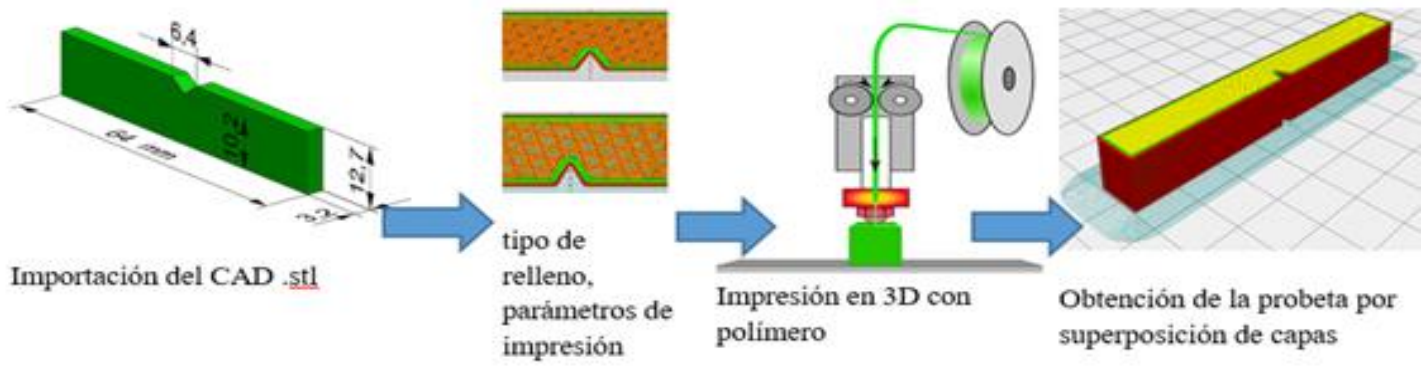

Fuente: Elaboración propia.

\section{Ensayos de impacto Izod}

Los ensayos de impacto Izod se realizaron en probetas impresas en 3D con filamento de ABS y PLA. Con las probetas estandarizadas se procede al desarrollo del ensayo de impacto Izod en función de la norma ASTM D256 como, como se muestra en la figura.

Figura 11. Ubicación de probetas para el ensayo IZOD.

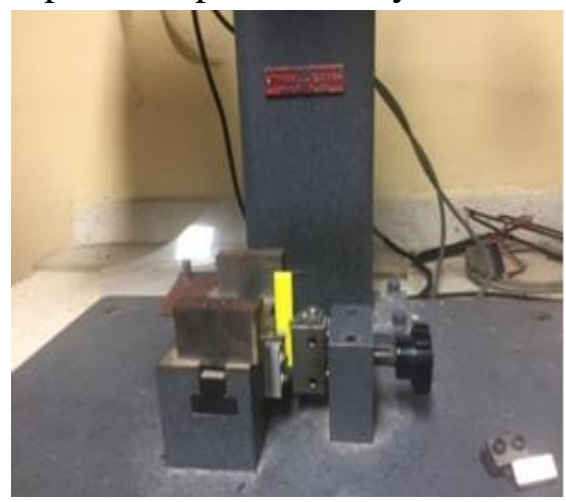

Fuente: Elaboración propia. 


\section{Ensayo de dureza tipo shore D en las probetas impresas en 3D.}

Se realizaron 10 mediadas de dureza shore D en cada una de las probetas de PLA y ABS. El proceso es muy sencillo, consiste en presionar el identador del durómetro en diferentes partes de la superficie de la probeta, en cada ensayo se tomó y registro datos de dureza Shore D. A simple vista se puede observar la superficie porosa de la probeta, por lo cual los valores de dureza son bajos, respecto a una probeta compactada con plástico fundido.

Figura 12. Ensayo dureza Shore D en probetas.

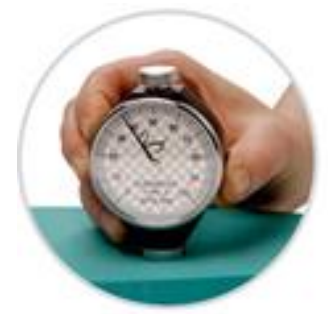

Fuente: Laboratorio Materiales ESPOCH

\section{Resultados}

Los resultados obtenidos de los ensayos de impacto tipo Izod realizados en probetas impresas en 3D, mediante ensayo de péndulo tipo Izod en función de los lineamientos de la norma ASTM D256, se tabulan a continuación considerando el material, tipo de estructura de relleno y el ancho de probeta. Las unidades de la energía de impacto se expresan en Joule/metro.

En la tabla 2, se tabulan los resultados para una probeta de PLA, estructura cubica y ancho de probeta de $13 \mathrm{~mm}$, el promedio de los ensayos de impacto, arroja una capacidad de absorción de energía de impacto $17.78 \mathrm{~J} / \mathrm{M}$.

Tabla 2. Energía de impacto Izod en probetas de PLA impresas en 3D con estructura cúbica

Probeta Impresa 3D: PLA

Estructura relleno: Cúbica

\begin{tabular}{cc}
\hline $\begin{array}{c}\text { Ancho de probeta } \\
(\mathbf{m m})\end{array}$ & $\begin{array}{c}\text { Energía Impacto Izod } \\
\mathbf{S 1}(\mathbf{J} / \mathbf{m})\end{array}$ \\
\hline 13 & 17,72 \\
13 & 17,46 \\
13 & 17,85 \\
13 & 17,59 \\
13 & 18,49 \\
13 & 17,55 \\
\hline $\mathbf{1 3}$ & $\mathbf{1 7 , 7 8}$ \\
\hline
\end{tabular}

Fuente: Elaboración propia. 
En la tabla 3, se tabulan los resultados para una probeta de PLA, estructura tri hexagonal y ancho de probeta de $12.6 \mathrm{~mm}$, el promedio de los ensayos de impacto, arroja una capacidad de absorción de energía de impacto 19.20J/M.

Tabla 3. Energía de impacto Izod en probetas de PLA impresas en 3D con estructura tri hexagonal.

Probeta Impresa 3D: PLA

Estructura relleno: Tri hexagonal

\begin{tabular}{cc}
\hline $\begin{array}{c}\text { Ancho de probeta } \\
(\mathbf{m m})\end{array}$ & $\begin{array}{c}\text { Energía Impacto Izod } \\
\mathbf{S 1}(\mathbf{J} / \mathbf{m})\end{array}$ \\
\hline 12,7 & 16,09 \\
12,5 & 20,11 \\
13 & 21,3 \\
12,5 & 21,31 \\
12,5 & 17,66 \\
12,5 & 18,7 \\
\hline 12,6 & 19,20 \\
\hline
\end{tabular}

Fuente: Elaboración propia.

En la tabla 4, se tabulan los resultados para una probeta de ABS, estructura cubica y ancho de probeta de $12.7 \mathrm{~mm}$, el promedio de los ensayos de impacto, arroja una capacidad de absorción de energía de impacto $38.50 \mathrm{~J} / \mathrm{M}$.

Tabla 4. Energía de impacto Izod en probetas de ABS impresas en 3D con estructura cúbica.

Probeta Impresa 3D: ABS

Estructura relleno: Cúbica

\begin{tabular}{cc}
\hline $\begin{array}{c}\text { Ancho de probeta } \\
(\mathbf{m m})\end{array}$ & $\begin{array}{c}\text { Energía Impacto Izod } \\
\mathbf{S 1}(\mathbf{J} / \mathbf{m})\end{array}$ \\
\hline 13 & 38,34 \\
13 & 37,47 \\
12,7 & 38,66 \\
12,5 & 39,69 \\
12,5 & 39,28 \\
13 & 37,58 \\
\hline 12,7 & 38,50 \\
\hline
\end{tabular}

Fuente: Elaboración propia.

En la tabla 5, se tabulan los resultados para una probeta de ABS, estructura tri hexagonal y ancho de probeta de $12.7 \mathrm{~mm}$, el promedio de los ensayos de impacto, arroja una capacidad de absorción de energía de impacto 34.91J/M. 
Tabla 5. Energía de impacto Izod en probetas de ABS impresas en 3D con estructura tri hexagonal.

Probeta Impresa 3D: ABS

Estructura relleno: Tri hexagonal

\begin{tabular}{cc}
\hline $\begin{array}{c}\text { Ancho de probeta } \\
(\mathbf{m m})\end{array}$ & $\begin{array}{c}\text { Energía Impacto Izod } \\
\mathbf{S 1}(\mathbf{J} / \mathbf{m})\end{array}$ \\
\hline 12,5 & 37,74 \\
13 & 33,85 \\
13 & 35,18 \\
13 & 32,14 \\
12,5 & 37,83 \\
12,7 & 32,69 \\
\hline 12,7 & 34,91 \\
\hline
\end{tabular}

Fuente: Elaboración propia.

De acuerdo con los datos que se muestran en las tablas anteriores se establece un gráfico comparativo entre los resultados obtenidos en los ensayos de impacto Izod en probetas de PLA y ABS, con respecto al tipo de estructura de relleno. En la siguiente gráfica se puede determinar que las probetas de ABS con estructura de relleno tipo cubica absorbe mayor energía de impacto; por lo que se considera una buena opción utilizar ABS en los productos impresos en 3D, se recomienda hacer ensayos de toxicidad y flamabilidad, por el motivo que los productos están en contacto con el usuario.

Figura 13. Energía de impacto Izod en probetas de PLA y ABS.

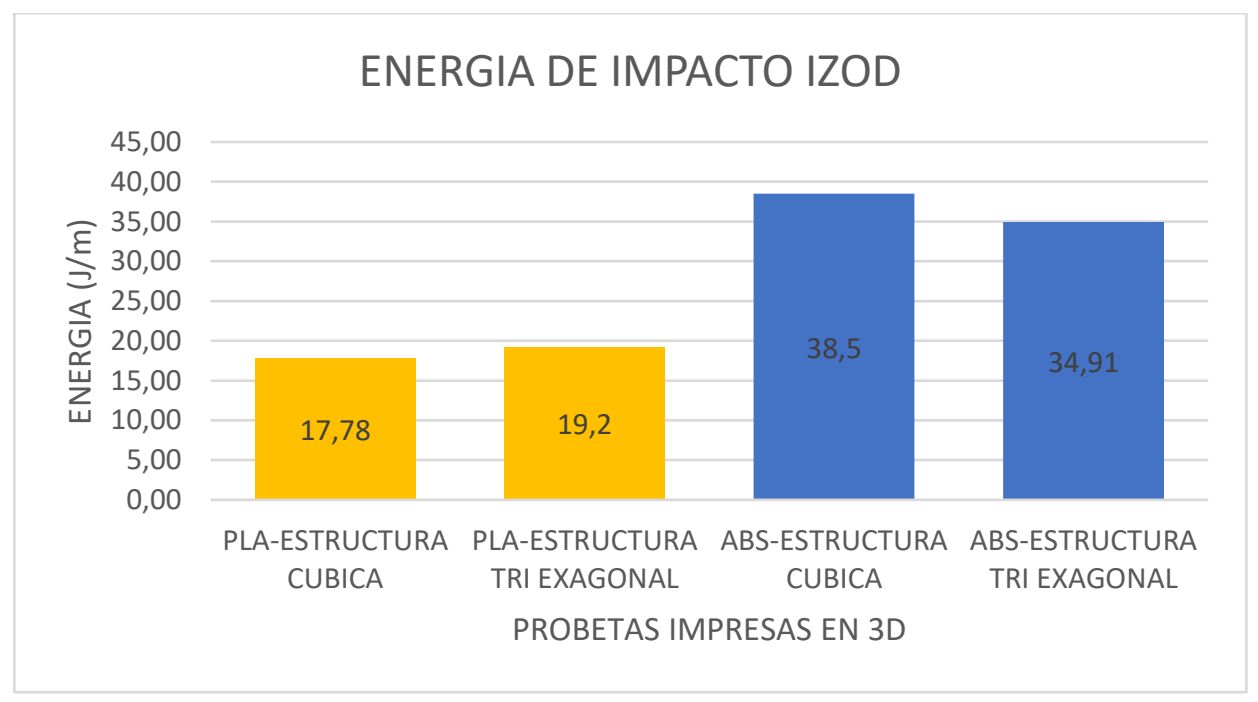

Fuente: Elaboración propia. 


\section{Microscopia de la superficie de fractura.}

Los resultados de los ensayos de microscopia SEM, muestran que la probeta de ABS con estructura de relleno cúbica de un $80 \%$ es mucho más compacto con respecto a la probeta PLA considerando que tiene igual tipo estructura. En la siguiente figura se puede observar los cordones de material y el tipo de relleno depositados por la máquina de prototipado, además se observa las grietas que se forman entre los niveles superpuestos. Debido a la falta de compactación del plástico, los productos impresos en 3D no se los utiliza en máquinas o equipos en donde los esfuerzos críticos son elevados. Generalmente los productos impresos en 3D nos ayudan a verificar forma, ergonómica, comodidad, entre otros; sin embargo cuando se trata de obtener órtesis personalizadas a bajo costo, este método de manufactura aditiva es la mejor alternativa.

Figura 14. SEM, superficies fracturadas por el método de impacto Izod en probetas impresas en 3D.

ABS estructura cúbica $80 \%$
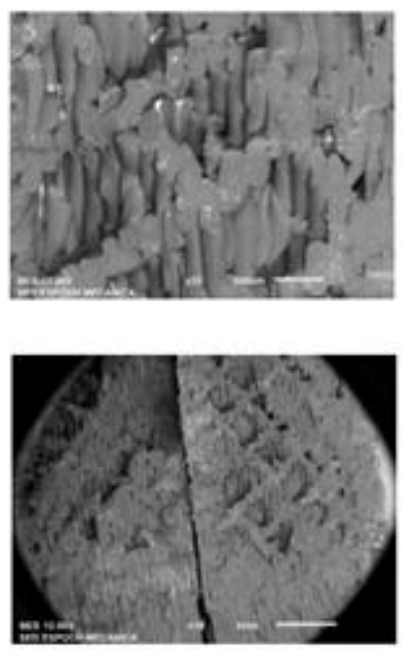

PLA estructura cúbica $80 \%$
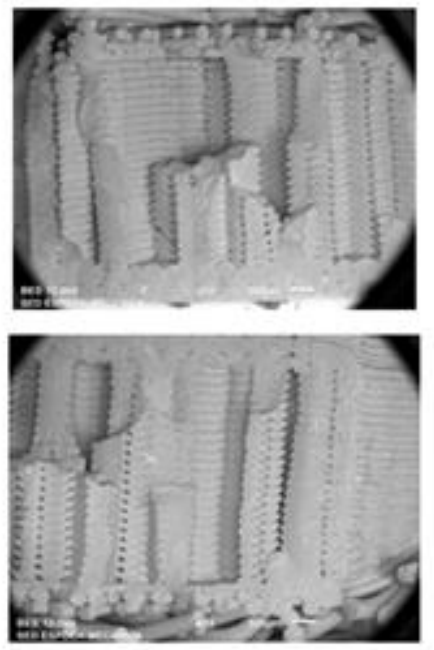

Fuente: Elaboración propia.

En la figura 15 se observa la propagación de la fisura a partir de un punto denominado muesca (forma de V) de probeta normalizada. Luego del ensayo de impacto Izod se procede a juntar las mitades de la probeta fracturada, mediante inspección visual se observa que la probeta de PLA con estructura de relleno cúbica muestra una rotura lineal uniforme (rotura frágil, se puede considerar que un polímero es frágil debido a la alta velocidad de aplicación de la carga de impacto), mientras que en la probeta de ABS con la misma estructura de relleno, se observa una propagación de la fisura con forma de zigzag. Es importante decir que la dirección de aporte del cordón de material fundido es longitudinal para todas las probetas ensayadas, si la dirección de 
impresión coincide con la dirección de la carga la resistencia mecánica de la probeta disminuye considerablemente; por lo que se recomienda tener en cuenta la dirección de acción de la carga, para configurar una dirección de impresión en 3D adecuado, generalmente se recomienda que la dirección de la carga sea perpendicular a la dirección de aporte del polímero fundido para obtener mayor resistencia mecánica.

Figura 15. Análisis de fractura en probetas fracturadas por el método de impacto Izod.
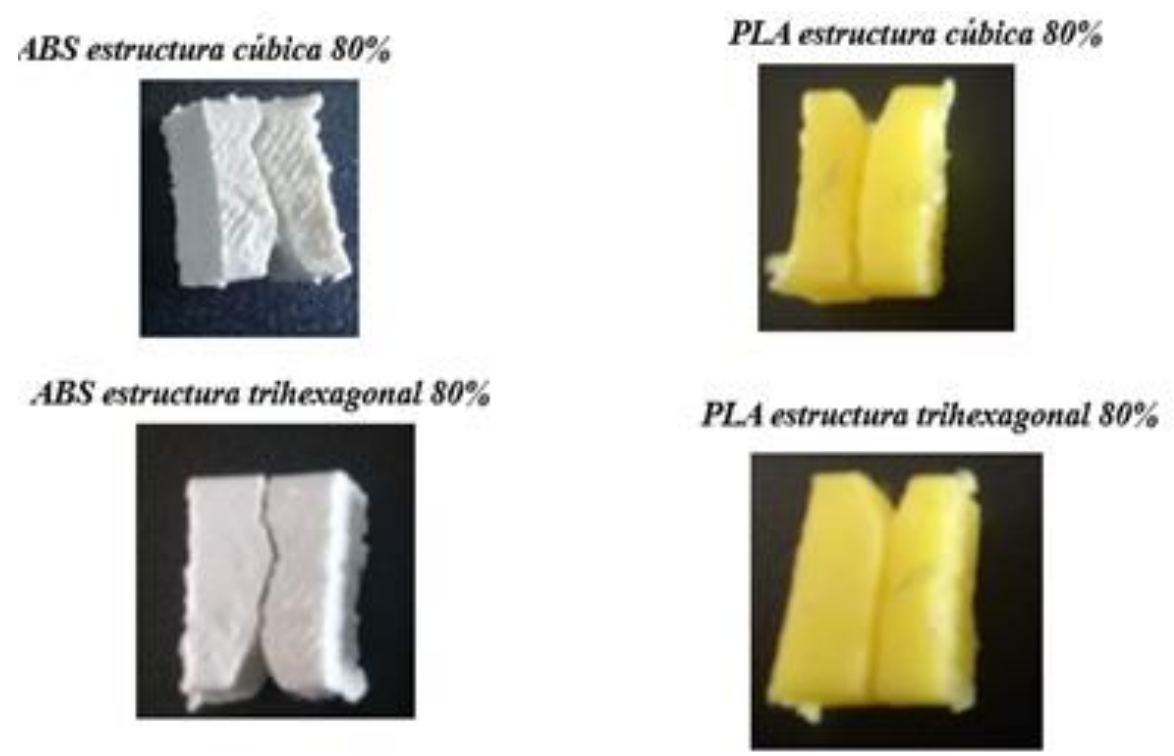

Fuente: Elaboración propia.

Ensayo de dureza Shore D en polímeros impresos en 3D.

Los resultados de los ensayos de dureza Shore D para termoplásticos en probetas impresas en 3D de ABS y PLA, consistió en tomar medidas de dureza en diferentes partes aleatorias de las probetas de ABS y PLA.

La dureza de las probetas impresas en 3D es notablemente menor comparado con respecto a durezas de probetas que fueron obtenidas mediante inyección de plástico; en función de los datos obtenidos se determina que las probetas de ABS es más difícil de penetrar con respecto al material PLA.

Mediante inspección visual se observa que la superficie externa de la probeta tiene porosidades que afectan a la dureza de la probeta, esto es debido a la facilidad de ingreso del identador del durómetro en las porosidades, esto genera incertidumbre en los datos tomados.

En la siguiente tabla se muestran los datos obtenidos del ensayo de dureza shore $\mathrm{D}$ en las probetas prototipadas. La toma de medidas de dureza se realizó en 10 puntos diferentes de cada probeta, considerando su material y tipo de estructura de relleno. 
Tabla 6. Ensayos de dureza Shore D en probetas impresas en 3D.

DUREZA SHORE D, EN PROBETAS IMPRESAS EN 3D, ABS Y PLA

\begin{tabular}{|c|c|c|c|c|}
\hline & ABS & & PLA & PIA \\
\hline PROBETA & $\begin{array}{c}\text { ESTRUCTURA } \\
\text { TRI } \\
\text { HEXAGONAL }\end{array}$ & $\begin{array}{c}\text { ESTRUCTURA } \\
\text { CUBICA }\end{array}$ & $\begin{array}{c}\text { ESTRUCTURA } \\
\text { TRI } \\
\text { EXAGONAL }\end{array}$ & $\begin{array}{c}\text { ESTRUCTURA } \\
\text { CUBICA }\end{array}$ \\
\hline 1 & 69.5 & 61 & 62. & 68 \\
\hline 2 & 60.5 & 65.5 & 64.5 & 60.5 \\
\hline 3 & 66 & 64.5 & 63.5 & 64 \\
\hline 4 & 67 & 62.5 & 61.5 & 63 \\
\hline 5 & 65 & 62 & 60.5 & 61.5 \\
\hline 6 & 65 & 61.5 & 60.5 & 63.5 \\
\hline 7 & 64.5 & 67.5 & 59. & 64 \\
\hline 8 & 68.5 & 62.5 & 61 & 62.5 \\
\hline 9 & 63.5 & 64.5 & 60 & 61.5 \\
\hline 10 & 67.5 & 61 & 61 & 60.5 \\
\hline Promedio & 65.8 & 61.3 & 60.7 & 64.8 \\
\hline
\end{tabular}

Fuente: Elaboración propia.

En base a la tabla anterior, se gráfica los resultados del ensayo de dureza Shore D, relacionando el tipo de material con respecto al tipo de estructura de relleno. A continuación se observa una gráfica comparativa, que permite determinar que la probeta de ABS y estructura tri hexagonal presenta mayor oposición a ser marcado.

Figura 16. Resultados ensayos de dureza Shore D.

\section{Dureza Shore D en termoplásticos}

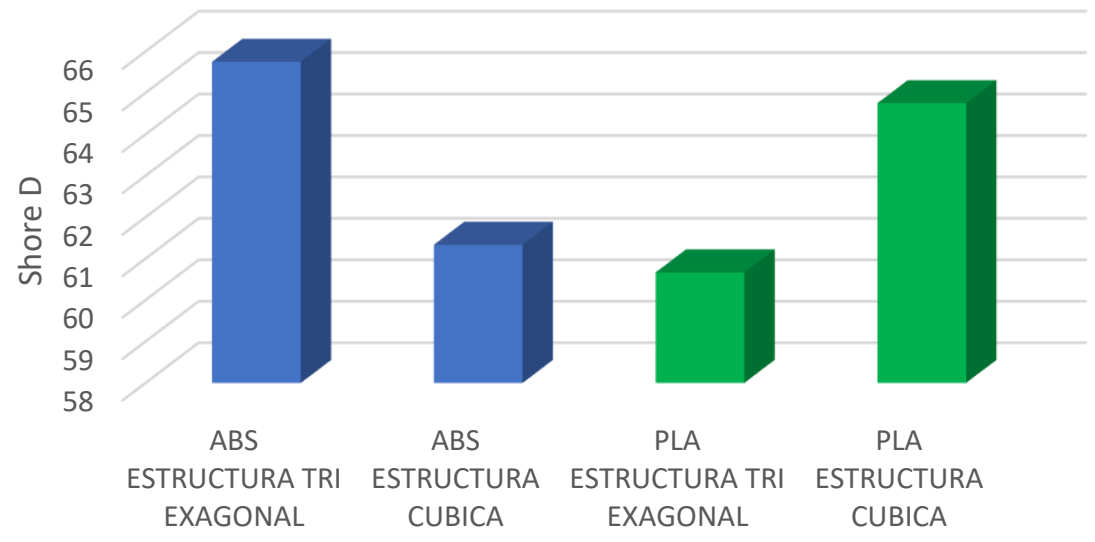

Fuente: Elaboración propia. 
Después de analizar los resultados de dureza en ABS y PLA, se determina que para efectos de prototipado con impresión 3D con filamento polímero, en donde la resistencia al desgaste o acabado superficial es importante, se considera una buena opción el filamento de ABS con una estructura tri hexagonal.

\section{Conclusiones.}

- De acuerdo al ensayo de impacto tipo IZOD aplicando la norma ASTM D256, se determina que la probeta impresa en 3D con material ABS y estructura cúbica, es la probeta que mayor cantidad de energía de impacto puede absorber, debido principalmente a la configuración de la geometría del material de relleno y su densidad.

- De acuerdo con los datos tabulados, considerando dos materiales y sus estructuras de relleno para el proceso, de donde se determina que el material ABS con malla de estructura cubica, absorbe una energía de impacto de $38,5 \mathrm{~J} / \mathrm{m}$.

- En el ensayo de dureza Shore tipo D para termoplásticos, se determina que la probeta de PLA impresa en 3D y con estructura cúbica tiene mayor dureza y está directamente relacionada con el tipo de estructura de relleno.

- Se determina que el material PLA tiene una dureza superior al ABS, por lo cual absorbe menos energía de impacto, por lo que se observa que la propagación de la fisura en el PLA es lineal.

- En función a las fotografías de la superficie de la fractura obtenidas en el microscopio (SEM), se determina que entre las capas de material fundido depositado por la máquina de impresión 3D, se generan espacios o grietas, que son potenciales focos para la propagación de la fisura.

\section{Referencias bibliográficas.}

Arce, G. C. (2005). Ortesis de miembros superiores. Clasificación, Funciones, Prototipos, Caracter $\{\backslash \backslash \backslash i\}$ sticas, Indicaciones [Internet]. Lima, Perú: Medicina de Rehabilitación.

Cardona, S. L., Grajales, D. H. M., \& Castro, W. P. (2016). Instrumentación de un péndulo tipo Izod, para evaluación de la resistencia al impacto de pol\{ $\backslash \backslash \backslash \mathrm{i}\}$ meros. Universidad Tecnológica de Pereira. Facultad de Tecnolog $\{\backslash ’ \mid$ \} $\}$ as. Tecnolog $\{\backslash ’ \mid i\} a$ Mecánica.

León, M., \& Marcos-Fernández, Á. (2019). Impresión 3D con materiales elástoméricos. Revista De Plásticos Modernos, 118(747).

Mix, A. W., \& Giacomin, A. J. (2011). Standardized polymer durometry. Journal of Testing and Evaluation, 39(4), 696-705.

Molina Osejos, J. V. (2016). Caracterización de materiales termoplásticos de ABS y PLA semir $\{\backslash \backslash \backslash i\}$ gido impresos en 3D con cinco mallados internos diferentes. Quito, 2016. 
Vol. 3, N², p. 17-33, abril-junio, 2020

Ramos, F. L. S., Mijares, E. M., \& Gudiño, P. O. (2015). Introducción a la ciencia de los pol $\{\backslash \backslash \backslash i\}$ meros. México.

Reyes, L. G. (n.d.). Análisis documental de las ventajas de la impresión 3D Documentary analysis of the advantages of 3D printing Análise documental das vantagens de impressão 3D.

Roberson, D. A., Perez, A. R. T., Shemelya, C. M., Rivera, A., MacDonald, E., \& Wicker, R. B. (2015). Comparison of stress concentrator fabrication for 3D printed polymeric izod impact test specimens. Additive Manufacturing, 7, 1-11.

Sin, L. T. (2012). Polylactic acid: PLA biopolymer technology and applications. William Andrew.

Torres, E., León, J., \& Torres, E. (2012). Diseño y construcción de una impresora 3D aplicando la técnica de prototipado rápido modelado por deposición fundida. Ponencia Llevada a Cabo En El Tercer Congreso Argentino de Ingenier $\{\backslash \backslash \backslash i\} a$ Mecánica, Buenos Aires, Argentina.

Tovar, V. A. C., Rl’lios, A. R., Cepeda, L. F., Galindo, A. S., \& Saltillo, C. (2018). Análisis de las propiedades fisicoqu $\{\backslash \backslash \mid \mathrm{i}\}$ micas de materiales poliméricos para re-uso en impresiones 3D. Macromolecules. 


\section{PARA CITAR EL ARTÍCULO INDEXADO.}

Escobar Guachambala, M. Ángel, Gavilanes Carrión, J. J. G. C., \& Freire Quintanilla, M. H. (2020). Evaluación de la capacidad de absorción de energía de impacto y dureza en probetas impresas en 3D de PLA y ABS con estructura cúbica y tri hexagonal. ConcienciaDigital, 3(2), 1733. https://doi.org/10.33262/concienciadigital.v3i2.1204

\section{\Ciencia}

El artículo que se publica es de exclusiva responsabilidad de los autores y no necesariamente reflejan el pensamiento de la Revista Ciencia Digital.

El artículo queda en propiedad de la revista y, por tanto, su publicación parcial y/o total en otro medio tiene que ser autorizado por el director de la Revista Ciencia Digital.
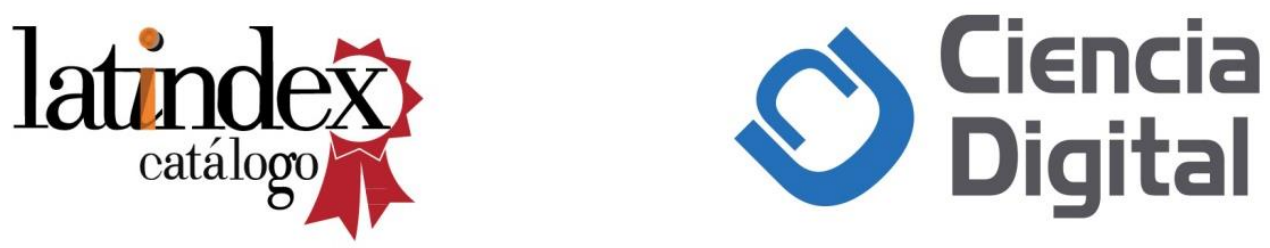